\title{
Gelfand-Tzetlin Coordinates for the Unitary Supergroup
}

\author{
Thomas Guhr ${ }^{\star}$ \\ Center for Chaos and Turbulence Studies, Niels Bohr Institute, Blegdamsvej 17, DK-2100 Copenhagen $\emptyset$, \\ Denmark
}

Received: 22 November 1994

\begin{abstract}
The Gelfand-Tzetlin method provides explicit coordinates on the parameter space of the unitary group $U(k)$ which make direct evaluations of group integrals possible. It is closely related to the Gelfand construction of finite-dimensional irreducible representations. We generalize the Gelfand-Tzetlin method to the unitary supergroup $U\left(k_{1} / k_{2}\right)$. The coordinates on the parameter space for supergroup integrals and the invariant Haar measure are evaluated. As an example, the supersymmetric Harish-Chandra-Itzykson-Zuber integral is calculated. A generalized Gelfand pattern containing anticommuting variables is introduced which determines the representation.
\end{abstract}

\section{Introduction}

Almost half a century ago, Gelfand [6] constructed representations of the unitary group based on a chain of unitary subgroups in which the dimension is lowered by one in every step. The advantage of using such a recursive embedding becomes obvious in the structure of the Gelfand patterns which determine these representations and results naturally in many applications in physics $[4,2]$. At the same time, Gelfand and Tzetlin $[8,7]$ constructed explicit coordinates on the parameter space of the unitary group which are the interpretation of the discrete integers in the Gelfand pattern as continuous variables. Hence, this so-called Gelfand-Tzetlin method is closely related to representation theory. Recently, Shatashvili [17] used the Gelfand-Tzetlin coordinates in order to evaluate the correlation functions in the Itzykson-Zuber model $[15,14]$. These calculations reflect the recursive structure of the Gelfand-Tzetlin method directly.

The purpose of this paper is the generalization of the Gelfand-Tzetlin method to the unitary supergroup. Berezin [3] found a nontrivial extension of the standard analysis by employing anticommuting degrees of freedom. He also extended the standard algebra and group theory by defining supermatrices and supergroups. After the pioneering work of Efetov [5], these tools are being used in many areas of physics [18]. Furthermore, a supersymmetric generalization of the Harish-Chandra-Itzykson-Zuber

\footnotetext{
* Present address: Max Planck Institut für Kernphysik, Saupfercheckweg 1, D-69117 Heidelberg, Germany, e-mail: guhr@mickey.mpi-hd.mpg.de
} 
integral was found [9] which turned out to be useful in the theory of random matrices $[13,12]$. Further applications of supersymmetry, however, call for explicit coordinates on the parameter spaces of unitary supergroups and Hermitian supermatrices. These requirements led to the work to be presented here. The Gelfand-Tzetlin method is generalized in Sect. 2. As an example, the supersymmetric version of the HarishChandra-Itzykson-Zuber integral is rederived in Sect. 3. In Sect. 4, some aspects of representation theory are discussed. Certain calculations are performed in the appendix.

The reader might find it unpleasant and difficult to fight his way through the forest of the rather complicated equations, richly decorated with indices, which are to come in the body and the appendix of the paper. The author would like to assure the reader who is mainly intertested in the purely mathematical aspects that honest attempts were undertaken to develop a less pedantic and more compact type of notation. Regrettably, they were not successful. Those, however, who want to employ all these formulae in explicit calculations will quickly realize, that, under the given circumstances, the notation used here is quite convenient.

\section{Generalized Gelfand-Tzetlin Method}

After formulating the problem in Sect. 2.1, we derive the Gelfand-Tzetlin equations in Sect. 2.2. In Sects. 2.3 and 2.4 we solve these equations for small and for arbitrary dimensions, respectively. The basis transformation is discussed in Sect. 2.5 and the invariant Haar measure is evaluated in Sect. 2.6. The recursion of the method to all levels is performed in Sect. 2.7 and, finally, in Sect. 2.8 all matrix elements and the total invariant measure are expressed in Gelfand-Tzetlin coordinates.

2.1. Formulation of the Problem. We consider Hermitian graded or super-matrices $\sigma$ of dimension $k_{1}+k_{2}$ which have the form

$$
\sigma=\left[\begin{array}{cc}
\sigma^{C 1} & \sigma^{A \dagger} \\
\sigma^{A} & i \sigma^{C 2}
\end{array}\right]
$$

where $\sigma^{C j}, j=1,2$ are ordinary Hermitian $k_{j} \times k_{j}$ matrices and $\sigma^{A}$ is a $k_{2} \times k_{1}$ matrix whose entries are anticommuting variables. This notation is referred to as the boson fermion block notation. The diagonalization reads

$$
\sigma=u^{-1} s u
$$

where $u$ is an element of the unitary supergroup $U\left(k_{1} / k_{2}\right)$ divided by the Cartan subgroup, i.e. by the product of $k_{1}+k_{2}$ groups $U(1)$. The diagonal matrix of the eigenvalues

$$
s=\operatorname{diag}\left(s_{11}, \ldots, s_{k_{1} 1}, i s_{12}, \ldots, i s_{k_{2} 2}\right)
$$

contains $k_{1}$ eigenvalues $s_{p 1}$ in the boson boson and $k_{2}$ eigenvalues $i s_{p 2}$ in the fermion fermion block. We use the notation $s_{p}=s_{p 1}, p=1, \ldots, k_{1}$ and $s_{p+k_{1}}=i s_{p 2}, p=$ $1, \ldots, k_{2}$. The transformation law for the Cartesian volume element

$$
d[\sigma]=\prod_{j=1}^{2} \prod_{p=1}^{k_{j}} d \sigma_{p p}^{C j} \prod_{p>q} d \operatorname{Re} \sigma_{p q}^{C j} d \operatorname{Im} \sigma_{p q}^{C j} \prod_{p, q} d \sigma_{p q}^{A *} \sigma_{p q}^{A}
$$


to eigenvalue-angle coordinates is easily calculated by using the methods of reference [9],

$$
d[\sigma]=B_{k_{1} k_{2}}^{2}(s) d[s] d \mu(u),
$$

where $d \mu(u)$ is the invariant Haar measure. The square root of the Berezinian is given by

$$
B_{k_{1} k_{2}}(s)=\frac{\prod_{p>q}^{k_{1}}\left(s_{p 1}-s_{q 1}\right) \prod_{p>q}^{k_{2}}\left(i s_{p 2}-i s_{q 2}\right)}{\prod_{p, q}\left(s_{p 1}-i s_{q 2}\right)} .
$$

In the special case $k_{2}=0$, we find the usual transformation law for ordinary Hermitian matrices, in particular the Berezinian reduces to the squared Vandermonde determinant,

$$
B_{k_{1}, 0}(s)=\Delta_{k_{1}}(s)=\prod_{p>q}\left(s_{p 1}-s_{q 1}\right)
$$

and similarly for $k_{1}=0, k_{2} \neq 0$. Moreover, in the special case $k_{1}=k_{2}$ the square root of the Berezinian acquires the structure of an ordinary determinant

$$
B_{k_{1} k_{1}}(s)=\operatorname{det}\left[\frac{1}{s_{p 1}-i s_{q 2}}\right]_{p, q=1, \ldots, k_{1}}
$$

reflecting the determinantal structure of the correlation functions of the Gaussian Unitary Ensemble [9].

Our goal is the explicit construction of a coordinate system on the space parametrized by the matrices $u$. This is done by a proper generalization of the GelfandTzetlin coordinates.

2.2. Gelfand-Tzetlin Equations. The original, more geometric method [7] to construct the Gelfand-Tzetlin equations for ordinary unitary matrices needs to be modified to a more algebraic procedure in the case of supermatrices for reasons which will become clear in the following. Define $u_{p}, p=1, \ldots, k_{1}+k_{2}$ as the columns of $u$ with entries $u_{q p}$. These vectors satisfy $u_{p}^{\dagger} u_{q}=\delta_{p q}$ and form an orthonormal basis of a $k_{1}+k_{2}$ dimensional space of complex supervectors. We can express the Haar measure in the form

$$
d \mu(u)=d \mu\left(u_{1}, \ldots, u_{k_{1}+k_{2}}\right)=d \mu\left(u_{1}\right) d \mu\left(u_{2}, \ldots, u_{k_{1}+k_{2}}\right) .
$$

Since $u_{1}$ is an unitvector, the number of independent variables is $2\left(k_{1}+k_{2}\right)-1$, and the elements of $u_{1}$ cannot be used directly as independent variables. The idea is to project onto the $k_{1}+k_{2}-1$ dimensional subspace spanned by the vectors $u_{2}, \ldots, u_{k_{1}+k_{2}}$. The corresponding projection $\left(1-u_{1} u_{1}^{\dagger}\right) s\left(1-u_{1} u_{1}^{\dagger}\right)$ of the eigenvalue matrix $s$ has rank $k_{1}+k_{2}-1$ and the eigenvalue equation

$$
s_{p}^{(1)} e_{p}^{(1)}=\left(1-u_{1} u_{1}^{\dagger}\right) s\left(1-u_{1} u_{1}^{\dagger}\right) e_{p}^{(1)}=\left(1-u_{1} u_{1}^{\dagger}\right) s e_{p}^{(1)}
$$

has $k_{1}+k_{2}-1$ solutions $s_{p}^{(1)}, p=2, \ldots, k_{1}+k_{2}$. We refer to them as the generalized Gelfand-Tzetlin eigenvalues. The basis spanned by the $k_{1}+k_{2}-1$ orthonormal eigenvectors $e_{p}^{(1)}$ is a rotation of the basis $u_{2}, \ldots, u_{k_{1}+k_{2}}$. We can view the above equation as a definition of new independent variables $s_{p}^{(1)}$ which contain the $k_{1}+k_{2}$ eigenvalues $s_{p}, p=1, \ldots, k_{1}+k_{2}$ as parameters. In order to solve for the eigenvalues we consider the characteristic function, i.e. the superdeterminant 


$$
\begin{aligned}
z\left(s_{p}^{(1)}\right) & =\operatorname{detg}\left(\left(1-u_{1} u_{1}^{\dagger}\right) s-s_{p}^{(1)}\right) \\
& =\operatorname{detg}\left(s-s_{p}^{(1)}\right) \operatorname{detg}\left(1-\left(s-s_{p}^{(1)}\right)^{-1} u_{1} u_{1}^{\dagger} s\right) \\
& =\operatorname{detg}\left(s-s_{p}^{(1)}\right)\left(1-u_{1}^{\dagger} \frac{s}{s-s_{p}^{(1)}} u_{1}\right) \\
z\left(s_{p}^{(1)}\right) & =-s_{p}^{(1)} \operatorname{detg}\left(s-s_{p}^{(1)}\right) u_{1}^{\dagger} \frac{1}{s-s_{p}^{(1)}} u_{1} .
\end{aligned}
$$

There are $k_{1}-1$ eigenvalues $s_{p}^{(1)}=s_{p 1}^{(1)}, p=2, \ldots, k_{1}$ in the boson boson block and $k_{2}$ eigenvalues $s_{k_{1}+p}^{(1)}=i s_{p 2}^{(1)}, p=1, \ldots, k_{2}$ in the fermion fermion block. The function $z\left(s_{p}^{(1)}\right)$ behaves differently for the two types and the above equation has to be discussed in the limits

$$
z\left(s_{p}^{(1)}\right) \longrightarrow \begin{cases}0 & \text { for } p=2, \ldots, k_{1} \\ \infty & \text { for } p=k_{1}+1, \ldots, k_{1}+k_{2}\end{cases}
$$

Together with the condition $u_{1}^{\dagger} u_{1}=1$ we have $k_{1}+k_{2}$ equations to solve for the $k_{1}+k_{2}$ quantities $\left|u_{p 1}\right|^{2}=u_{p 1}^{*} u_{p 1}$. It is useful to write the commuting and anticommuting variables in the form $u_{p 1}=v_{p}^{(1)}, p=1, \ldots, k_{1}$ and $u_{\left(k_{1}+p\right) 1}=\alpha_{p}^{(1)}, p=1, \ldots, k_{2}$, respectively, and to introduce the corresponding $k_{1}$ and $k_{2}$ component vectors $v^{(1)}$ and $\alpha^{(1)}$. The $k_{1}+k_{2}$ equations read then explicitly

$$
\begin{aligned}
& 1=\sum_{p=1}^{k_{1}}\left|v_{p}^{(1)}\right|^{2}+\sum_{p=1}^{k_{2}}\left|\alpha_{p}^{(1)}\right|^{2}, \\
& 0=\sum_{q=1}^{k_{1}} \frac{\left|v_{q}^{(1)}\right|^{2}}{s_{q 1}-s_{p 1}^{(1)}}+\sum_{q=1}^{k_{2}} \frac{\left|\alpha_{q}^{(1)}\right|^{2}}{i s_{q 2}-s_{p 1}^{(1)}}, \quad p=2, \ldots, k_{1}, \\
& z_{p}=i s_{p 2}^{(1)} \frac{\prod_{q=1}^{k_{1}}\left(s_{q 1}-i s_{p 2}^{(1)}\right)}{\prod_{q=1}^{k_{2}}\left(i s_{q 2}-i s_{p 2}^{(1)}\right)}\left(\sum_{q=1}^{k_{1}} \frac{\left|v_{q}^{(1)}\right|^{2}}{s_{q 1}-i s_{p 2}^{(1)}}+\sum_{q=1}^{k_{2}} \frac{\left|\alpha_{q}^{(1)}\right|^{2}}{i s_{q 2}-i s_{p 2}^{(1)}}\right), \\
& z_{p} \rightarrow \infty, p=1, \ldots, k_{2},
\end{aligned}
$$

which reduce to the ordinary Gelfand-Tzetlin equations in the case $k_{2}=0$. The occurrence of the new equations involving the limits $z_{p} \rightarrow \infty$ is the reason why the original way to derive the Gelfand-Tzetlin equations in the ordinary case had to be modified.

2.3. Solutions for Small Dimensions. The structure of Eqs. (2.13) leads to some singularities that have to be discussed carefully. To do this we first treat the case of small $k_{1}, k_{2}$ before we turn to arbitrary dimensions. This is also especially helpful for an understanding of the role of the anticommuting variables.

In the case $k_{1}=k_{2}=1$ there is only one Gelfand-Tzetlin eigenvalue $i s_{12}^{(1)}$ and Eqs. (2.13) reduce to 


$$
1=\left|v_{1}^{(1)}\right|^{2}+\left|\alpha_{1}^{(1)}\right|^{2} \quad \text { and } \quad z_{1}=i s_{12}^{(1)} \frac{s_{11}-i s_{12}^{(1)}}{i s_{12}-i s_{12}^{(1)}}\left(\frac{\left|v_{1}^{(1)}\right|^{2}}{s_{11}-i s_{12}^{(1)}}+\frac{\left|\alpha_{1}^{(1)}\right|^{2}}{i s_{12}-i s_{12}^{(1)}}\right)
$$

which yields after elimination of $\left|v_{1}^{(1)}\right|^{2}$

$$
z_{1}=\frac{i s_{12}^{(1)}}{i s_{12}-i s_{12}^{(1)}}\left(1+\frac{s_{11}-i s_{12}}{i s_{12}-i s_{12}^{(1)}}\left|\alpha_{1}^{(1)}\right|^{2}\right)
$$

To take the limit $z_{1} \rightarrow \infty$ this equation has to be inverted

$$
\frac{i s_{12}^{(1)}}{z_{1}}=i s_{12}-i s_{12}^{(1)}-\left(s_{11}-i s_{12}\right)\left|\alpha_{1}^{(1)}\right|^{2}
$$

and we find for the Gelfand-Tzetlin eigenvalue

$$
i s_{12}^{(1)}=i s_{12}-\left(s_{11}-i s_{12}\right)\left|\alpha_{1}^{(1)}\right|^{2} .
$$

The nontrivial part is the absolute value squared of an anticommuting variable. We introduce the complex anticommuting variable $\xi_{1}^{(1)}$ as the Gelfand-Tzetlin coordinate, i.e. as an independent variable,

$$
\left|\xi_{1}^{(1)}\right|^{2}=i s_{12}^{(1)}-i s_{12}=-\left(s_{11}-i s_{12}\right)\left|\alpha_{1}^{(1)}\right|^{2}
$$

The modulus squared of the vector elements can be expressed in the form

$$
\left|v_{1}^{(1)}\right|^{2}=+\frac{i s_{12}-s_{11}}{i s_{12}^{(1)}-s_{11}} \text { or }\left|\alpha_{1}^{(1)}\right|^{2}=-\frac{i s_{12}^{(1)}-i s_{12}}{s_{11}-i s_{12}} \text {. }
$$

Strictly speaking, the second of Eqs. (2.14) is ill-defined because it contains $i s_{12}^{(1)}-$ $i s_{12}=\left|\xi_{1}^{(1)}\right|^{2}$ in the denominator. To make the derivation mathematically cleaner, the projector in Eq. (2.10) has to be replaced by $1+\varepsilon-u_{1} u_{1}^{\dagger}$ with an infinitesimal increment $\varepsilon$. The Gelfand-Tzetlin equations are then well-defined and the eigenvalue is a function of $\varepsilon$. The limit $\varepsilon \rightarrow 0$ gives the result (2.17). Since this procedure is tedious but straightforward for arbitrary dimensions, we do not use it in the remainder. However, it has to be understood that all singularities of this type have to be treated in this way. When using expressions like $i s_{12}^{(1)}-i s_{12}=\left|\xi_{1}^{(1)}\right|^{2}$ in the denominator as a short-hand notation throughout the paper we simply mean that in the numerator the term $i s_{12}^{(1)}-i s_{12}=\left|\xi_{1}^{(1)}\right|^{2}$ has to be omitted.

Because of its special importance for Random Matrix Theory and in order to give an intuition for the structure of the general solution, we also discuss the case $k_{1}=k_{2}=2$ explicitly in App. A.

2.4. Solutions for Arbitrary Dimensions. The form of Eqs. (2.13) indicates the structure of the solutions for arbitrary values of $k_{1}$ and $k_{2}$, 


$$
\begin{aligned}
&\left|v_{p}^{(1)}\right|^{2}=\frac{\prod_{q=2}^{k_{1}}\left(s_{p 1}-s_{q 1}^{(1)}\right) \prod_{q=1}^{k_{2}}\left(s_{p 1}-i s_{q 2}\right)}{\prod_{q=1}^{k_{2}}\left(s_{p 1}-i s_{q 2}^{(1)}\right) \prod_{q=1, q \neq p}^{k_{1}}\left(s_{p 1}-s_{q 1}\right)}, \quad p=1, \ldots, k_{1}, \\
&\left|\alpha_{p}^{(1)}\right|^{2}=\left(i s_{p 2}-i s_{p 2}^{(1)}\right) \frac{\prod_{q=2}^{k_{1}}\left(i s_{p 2}-s_{q 1}^{(1)}\right) \prod_{q=1, q \neq p}^{k_{2}}\left(i s_{p 2}-i s_{q 2}\right)}{\prod_{q=1, q \neq p}^{k_{2}}\left(i s_{p 2}-i s_{q 2}^{(1)}\right) \prod_{q=1}^{k_{1}}\left(i s_{p 2}-s_{q 1}\right)}, \\
& p=1, \ldots, k_{2} .
\end{aligned}
$$

The moduli squared $\left|v_{p}^{(1)}\right|^{2}$ and $\left|\alpha_{p}^{(1)}\right|^{2}$ contain all differences of eigenvalues involving $s_{p 1}$ and $i s_{p 2}$, respectively. For $k_{2}=0$ we find the original Gelfand-Tzetlin result. Again, the form of the second of the above equations suggests the introduction of the anticommuting Gelfand-Tzetlin coordinates $\xi_{p}^{(1)}$ whose moduli squared are given by

$$
\left|\xi_{p}^{(1)}\right|^{2}=i s_{p 2}^{(1)}-i s_{p 2}, \quad p=1, \ldots, k_{2} .
$$

Equations (2.20) can be derived along the lines given for the case of smaller dimensions in the last subsection. This somewhat lengthy calculation is sketched in App. B. A more direct way to derive the formulae (2.20) involving the representation theory of supergroups should exist but could not be found.

2.5. Basis Transformation. As discussed in Sect. 2.2, the eigenvalue equation (2.10) is a rotation of the basis of the subspace spanned by the vectors $u_{p}, p=2, \ldots, k_{1}+k_{2}$ to a new basis spanned by the eigenvectors $e_{p}^{(1)}, p=2, \ldots k_{1}+k_{2}$. The first $k_{1}$ components of the vectors $u_{p}, p=1, \ldots, k_{1}$ and $e_{p}^{(1)}, p=2, \ldots, k_{1}$ are commuting, the last $k_{2}$ are anticommuting. In case of the vectors $u_{p}, e_{p}^{(1)}, p=k_{1}+1, \ldots, k_{1}+k_{2}$ it is the other way around. This reflects the existence of the two types of eigenvalues in the boson boson and the fermion fermion block. We also use the notation $e_{p 1}^{(1)}=e_{p}^{(1)}, p=2, \ldots, k_{1}$ and $e_{p 2}^{(1)}=e_{k_{1}+p}^{(1)}, p=1, \ldots, k_{2}$. Since we have $u_{1}^{\dagger} e_{p}^{(1)}=0$ by construction, the vectors $u_{1}, e_{2}^{(1)}, \ldots, e_{k_{1}+k_{2}}^{(1)}$ form a basis of the full space. To study the basis rotation further, we rewrite Eq. (2.10),

$$
s e_{p}^{(1)}=s_{p}^{(1)} e_{p}^{(1)}+u_{1} b_{p}^{(1)} \quad \text { where } \quad b_{p}^{(1)}=u_{1}^{\dagger} s e_{p}^{(1)}, \quad p=2, \ldots, k_{1}+k_{2},
$$

which can be viewed as an expansion of $s e_{p}^{(1)}$ in the full space and in the rotated basis, the crucial quantity is the expansion coefficient $b_{p}^{(1)}$. There is a Pythagoras type of theorem,

$$
\left|s e_{p}^{(1)}\right|^{2}=\left|s_{p}^{(1)}\right|^{2}+\left|b_{p}^{(1)}\right|^{2}
$$

We introduce the elementary basis $e_{p}, p=1, \ldots, k_{1}+k_{2}$, where $e_{p}$ has unity at the $p^{\text {th }}$ position and zeros elsewhere. Multiplication of Eq. (2.22) with $e_{q}^{\dagger}$ yields

$$
\left(s_{q}-s_{p}^{(1)}\right) e_{q}^{\dagger} e_{p}^{(1)}=u_{q 1} b_{p}^{(1)} .
$$

This expression exhibits a high symmetry between the role of the variables $s_{q}$ and $u_{q 1}$ on the one and $s_{p}^{(1)}$ and $b_{p}^{(1)}$ on the other side. The $k_{1}+k_{2}-1$ component vector $b^{(1)}$ and the $k_{1}+k_{2}$ component vector $u_{1}$ are in a way dual to each other. Therefore we can construct the variables $b_{p}^{(1)}$ from the formulae $(2.20)$ by interchanging the role of the eigenvalues $s_{p}, p=1, \ldots, k_{1}+k_{2}$ and $s_{p}^{(1)}, p=2, \ldots, k_{1}+k_{2}$. According to 
the structure of the eigenvectors, $b_{p}^{(1)}$ can be commuting or anticommuting, we write $w_{p}^{(1)}=b_{p}^{(1)}, p=2, \ldots, k_{1}$ for the commuting and $\beta_{p}^{(1)}=b_{k_{1}+p}^{(1)}, p=1, \ldots, k_{2}$ for the anticommuting ones. We arrive at

$$
\begin{aligned}
&\left|w_{p}^{(1)}\right|^{2}=\frac{\prod_{q=1}^{k_{1}}\left(s_{p 1}^{(1)}-s_{q 1}\right) \prod_{q=1}^{k_{2}}\left(s_{p 1}^{(1)}-i s_{q 2}^{(1)}\right)}{\prod_{q=1}^{k_{2}}\left(s_{p 1}^{(1)}-i s_{q 2}\right) \prod_{q=2, q \neq p}^{k_{1}}\left(s_{p 1}^{(1)}-s_{q 1}^{(1)}\right)}, \quad p=2, \ldots, k_{1} \\
&\left|\beta_{p}^{(1)}\right|^{2}=\left(i s_{p 2}^{(1)}-i s_{p 2}\right) \frac{\prod_{q=1}^{k_{1}}\left(i s_{p 2}^{(1)}-s_{q 1}\right) \prod_{q=1, q \neq p}^{k_{2}}\left(i s_{p 2}^{(1)}-i s_{q 2}^{(1)}\right)}{\prod_{q=1, q \neq p}^{k_{2}}\left(i s_{p 2}^{(1)}-i s_{q 2}\right) \prod_{q=2}^{k_{1}}\left(i s_{p 2}^{(1)}-s_{q 1}^{(1)}\right)} \\
& p=1, \ldots, k_{2}
\end{aligned}
$$

which of course can also be derived in a procedure similar to the one described in Sects. 2.3 and 2.4. Notice that the presence of the term $i s_{p 2}^{(1)}-i s_{p 2}=\left|\xi_{p}^{(1)}\right|^{2}$ allows to replace all eigenvalues $i s_{p 2}^{(1)}$ with $i s_{p 2}$ in the formula for $\left|\beta_{p}^{(1)}\right|^{2}$. With these results and with the expression

$$
e_{p}^{(1)}=\frac{1}{s-s_{p}^{(1)}} u_{1} b_{p}^{(1)}, \quad p=2, \ldots, k_{1}+k_{2},
$$

which follows from Eq. (2.24) we have an explicit formula for the eigenvectors.

The basis transformation from the $k_{1}+k_{2}$ dimensional space to the $k_{1}+k_{2}-1$ dimensional space can be written with a $\left(k_{1}+k_{2}-1\right) \times\left(k_{1}+k_{2}\right)$ dimensional matrix $B$ whose rows are the adjoint eigenvectors, $e_{p}^{(1) \dagger}, p=2, \ldots, k_{1}+k_{2}$. By construction, $B B^{\dagger}$ is the unit matrix in the $k_{1}+k_{2}-1$ dimensional space and $B^{\dagger} B$ is the projection operator $1-u_{1} u_{1}^{\dagger}$. The matrix

$$
\widehat{S}^{(1)}=B s^{(1)} B^{\dagger}=B s B^{\dagger}
$$

is a $\left(k_{1}+k_{2}-1\right) \times\left(k_{1}+k_{2}-1\right)$ dimensional matrix of the Gelfand-Tzetlin eigenvalues. Equation (2.24) can then be written in the form

$$
B s-\widehat{s}^{(1)} B=b^{(1)} u_{1}^{\dagger}
$$

which emphasizes the relation between the vector $b^{(1)}$ and the basis transformation.

2.6. Invariant Measure. We calculate the invariant Haar measure $d \mu\left(u_{1}\right)$ which was defined in Eq. (2.9) by evaluating the invariant length element squared,

$$
d u_{1}^{\dagger} d u_{1}=\sum_{p=1}^{k_{1}} d v_{p}^{(1) *} d v_{p}^{(1)}+\sum_{p=1}^{k_{2}} d \alpha_{p}^{(1) *} d \alpha_{p}^{(1)} .
$$

For the commuting vector elements we introduce polar coordinates

$$
v_{p}^{(1)}=\sqrt{\left|v_{p}^{(1)}\right|^{2}} \exp \left(i \vartheta_{p}^{(1)}\right), \quad p=1, \ldots, k_{1}
$$

and find for the differentials 


$$
d v_{p}^{(1) *} d v_{p}^{(1)}=\frac{\left(d\left|v_{p}^{(1)}\right|^{2}\right)^{2}}{4\left|v_{p}^{(1)}\right|^{2}}+\left|v_{p}^{(1)}\right|^{2}\left(d \vartheta_{p}^{(1)}\right)^{2} .
$$

The new independent variables are the Gelfand-Tzetlin eigenvalues in the boson boson block, the phase angles introduced above and the Gelfand-Tzetlin eigenvalues in the fermion fermion block, we use the ordering

$$
s_{21}^{(1)}, \ldots, s_{k_{1}}^{(1)}, \vartheta_{1}^{(1)}, \ldots, \vartheta_{k_{1}}^{(1)}, \xi_{1}^{(1)}, \xi_{1}^{(1) *}, \ldots, \xi_{k_{2}}^{(1)}, \xi_{k_{2}}^{(1) *} .
$$

After an explicit computation of the differentials of the vector elements and some further algebra which is sketched in App. $\mathrm{C}$ we can write the invariant length element squared in the form

$$
d u_{1}^{\dagger} d u_{1}=\frac{1}{4} \sum_{p=2}^{k_{1}} \frac{\left(d s_{p 1}^{(1)}\right)^{2}}{\left|w_{p}^{(1)}\right|^{2}}+\sum_{p=1}^{k_{1}}\left|v_{p}^{(1)}\right|^{2}\left(d \vartheta_{p}^{(1)}\right)^{2}+\sum_{p=1}^{k_{2}} \sqrt{\frac{\left|\alpha_{p}^{(1)}\right|^{2}}{\left|\beta_{p}^{(1)}\right|^{2}}} d \xi_{p}^{(1) *} d \xi_{p}^{(1)}
$$

The metric tensor for the new coordinates in the ordering (2.32) follows immediately from the invariant length element squared,

$$
\begin{aligned}
& g^{(1)}=\operatorname{diag}\left(g_{1}^{(1)}, g_{2}^{(1)}\right) \text {, } \\
& g_{1}^{(1)}=\operatorname{diag}\left(\frac{1}{4\left|w_{2}^{(1)}\right|^{2}}, \ldots, \frac{1}{4\left|w_{k_{1}}^{(1)}\right|^{2}},\left|v_{1}^{(1)}\right|^{2}, \ldots,\left|v_{k_{1}}^{(1)}\right|^{2}\right) \text {, } \\
& g_{2}^{(1)}=\operatorname{diag}\left(\frac{1}{2} \sqrt{\frac{\left|\alpha_{1}^{(1)}\right|^{2}}{\left|\beta_{1}^{(1)}\right|^{2}}}, \ldots, \frac{1}{2} \sqrt{\frac{\left|\alpha_{k_{2}}^{(1)}\right|^{2}}{\left|\beta_{k_{2}}^{(1)}\right|^{2}}}\right) \otimes\left[\begin{array}{cc}
0 & +1 \\
-1 & 0
\end{array}\right] \text {. }
\end{aligned}
$$

It should be emphasized that the metric tensor is diagonal in the commuting variables $s_{p 1}^{(1)}$ and $\vartheta_{p}^{(1)}$ and diagonal in the pairs $\xi_{p}^{(1)}, \xi_{p}^{(1) *}$ of anticommuting variables. This highly welcome and appreciated feature will have far reaching consequences for the harmonic analysis. Of course, the calculation of the invariant measure becomes trivial. From the superdeterminant of the metric tensor,

$$
\operatorname{detg} g^{(1)}=\frac{\prod_{p=1}^{k_{1}}\left|v_{p}^{(1)}\right|^{2} \prod_{p=1}^{k_{2}} 4\left|\beta_{p}^{(1)}\right|^{2}}{\prod_{p=2}^{k_{1}} 4\left|w_{p}^{(1)}\right|^{2} \prod_{p=1}^{k_{2}}\left|\alpha_{p}^{(1)}\right|^{2}}=4^{k_{2}-k_{1}+1} \frac{B_{\left(k_{1}-1\right) k_{2}}^{2}\left(s^{(1)}\right)}{B_{k_{1} k_{2}}^{2}(s)}
$$

we obtain for the invariant measure

$$
d \mu\left(u_{1}\right)=2^{k_{2}-k_{1}+1} \frac{B_{\left(k_{1}-1\right) k_{2}}\left(s^{(1)}\right)}{B_{k_{1} k_{2}}(s)} d\left[s_{1}^{(1)}\right] d\left[\vartheta^{(1)}\right] d\left[\xi^{(1)}\right],
$$

where we use the notation

$$
d\left[s_{1}^{(1)}\right]=\prod_{p=2}^{k_{1}} d s_{p 1}^{(1)}, \quad d\left[\vartheta^{(1)}\right]=\prod_{p=1}^{k_{1}} d \vartheta_{p}^{(1)} \quad \text { and } \quad d\left[\xi^{(1)}\right]=\prod_{p=1}^{k_{2}} d \xi_{p}^{(1) *} d \xi_{p}^{(1)}
$$

for the products of the differentials. The domain of integration for the commuting variables $s_{p 1}^{(1)}, p=2, \ldots, k_{1}$ follows analogously to the ordinary case from Eqs. (2.20) because the moduli squared of the vector elements are positive. Hence we have 


$$
\begin{gathered}
s_{(p-1) 1} \leq s_{p 1}^{(1)} \leq s_{p 1}, \quad p=2, \ldots, k_{1}, \\
0 \leq \vartheta_{p}^{(1)}<2 \pi, \quad p=1, \ldots, k_{1},
\end{gathered}
$$

and, of course, no bounds on the domain of integration for the anticommuting variables.

The invariant measure is a generalization of the original Gelfand-Tzetlin measure which is a ratio of Vandermonde determinants, it follows from formula (2.36) in the case $k_{2}=0$. This structure of the invariant measure is no surprise from the viewpoint of representation theory. It is known that the invariant measure of the unitary group can be found from the one of the Hermitian matrices by replacing the eigenvalues with the phases of the Cartan subgroup. Since the Gelfand-Tzetlin eigenvalues parametrize the reduction of an unitary vector space by one dimension, the invariant measure has to be the ratio of the invariant measures of the reduced and the original space. Apparently, these considerations do also apply to the unitary supergroup.

2.7. Recursion. The process of slicing off the column $u_{1}$ of the matrix $u$ by projecting onto a subspace can be continued recursively. At each step a basis transformation of the type discussed in Sect. 2.5 reduces the relevant space by one dimension. After $k_{1}$ steps all anticommuting variables are treated and we are left with the original Gelfand-Tzetlin problem for the ordinary unitary group $U\left(k_{2}\right)$. Since the procedure is rather straightforward, we simply summarize the results in the following. There are all together $k_{1}+k_{2}$ steps and corresponding subspaces defined through the projection operators

$$
p^{(m)}=1-\sum_{p=1}^{m} u_{p} u_{p}^{\dagger}, \quad m=1, \ldots, k_{1}+k_{2} .
$$

This expression is equivalent to

$$
p^{(m-1)}=\sum_{p=m}^{k_{1}+k_{2}} u_{p} u_{p}^{\dagger}=u_{m} u_{m}^{\dagger}+p^{(m)}, \quad m=1, \ldots, k_{1}+k_{2},
$$

where we include the zeroth level and $p^{(0)}=1$ for notational purposes. The projections read

$$
s^{(m)}=p^{(m)} s^{(m-1)} p^{(m)}, \quad m=1, \ldots, k_{1}+k_{2}
$$

with $s^{(0)}=s$. The Gelfand-Tzetlin equation at each level,

$$
s_{p}^{(m)} e_{p}^{(m)}=s^{(m)} e_{p}^{(m)}=p^{(m)} s^{(m-1)} p^{(m)} e_{p}^{(m)}
$$

has $k_{1}+k_{2}-m$ eigenvalues $s_{p}^{(m)}, p=m+1, \ldots, k_{1}+k_{2}$ and as many orthonormal eigenvectors $e_{p}^{(m)}, p=m+1, \ldots, k_{1}+k_{2}$. We write the elementary basis as $e_{p}=e_{p}^{(0)}$. The projection operators can be expressed as

$$
p^{(m)}=\sum_{p=m+1}^{k_{1}+k_{2}} e_{p}^{(m)} e_{p}^{(m) \dagger}
$$

since by construction we have

$$
e_{q}^{(m)^{\dagger}} u_{p}=0, \quad p=1, \ldots, m, q=m+1, \ldots, k_{1}+k_{2} .
$$


For the basis transformation at each step we need the expansion coefficients $b_{p}^{(m+1)}, p=$ $m+2, \ldots, k_{1}+k_{2}$ defined through the equations

$$
\begin{aligned}
s^{(m-1)} e_{p}^{(m)} & =s_{p}^{(m)} e_{p}^{(m)}+u_{m} b_{p}^{(m)} \\
b_{p}^{(m)} & =u_{m}^{\dagger} s^{(m-1)} e_{p}^{(m)}
\end{aligned}
$$

and the scalar products between the eigenvectors at subsequent steps which can be written as

$$
e_{q}^{(m-1) \dagger} e_{p}^{(m)}=\frac{e_{q}^{(m-1) \dagger} u_{m}}{s_{q}^{(m-1)}-s_{p}^{(m)}} b_{p}^{(m)}
$$

by using Eq. (2.45). Everything is now expressed as a function of the scalar products $e_{p}^{(m-1) \dagger} u_{m}$ and of the expansion coefficients $b_{p}^{(m)}$. These are the crucial quantities at all levels.

At the first $k_{1}$ levels, labeled $m=1, \ldots, k_{1}$, there are $k_{1}-m$ eigenvalues $s_{p 1}^{(m)}=$ $s_{p}^{(m)}, p=m+1, \ldots, k_{1}$ in the boson boson and $k_{2}$ eigenvalues $i s_{p 2}^{(m)}=s_{k_{1}+p}^{(m)}, p=$ $1, \ldots, k_{2}$ in the fermion fermion block. Correspondingly, we have $k_{1}-m$ eigenvectors $e_{p 1}^{(m)}=e_{p}^{(m)}, p=m+1, \ldots, k_{1}$ in the boson boson block with scalar products

$$
\begin{aligned}
& e_{p 1}^{(m-1) \dagger} u_{m}=\sqrt{\left|v_{p}^{(m)}\right|^{2}} \exp \left(i \vartheta_{p}^{(m)}\right), \\
&\left|v_{p}^{(m)}\right|^{2}= \frac{\prod_{q=m+1}^{k_{1}}\left(s_{p 1}^{(m-1)}-s_{q 1}^{(m)}\right) \prod_{q=1}^{k_{2}}\left(s_{p 1}^{(m-1)}-i s_{q 2}^{(m-1)}\right)}{\prod_{q=1}^{k_{2}}\left(s_{p 1}^{(m-1)}-i s_{q 2}^{(m)}\right) \prod_{q=m, q \neq p}^{k_{1}}\left(s_{p 1}^{(m-1)}-s_{q 1}^{(m-1)}\right)}, \\
& p=m, \ldots, k_{1},
\end{aligned}
$$

and $k_{2}$ eigenvectors $e_{p 2}^{(m)}=e_{k_{1}+p}^{(m)}, p=1, \ldots, k_{2}$ in the fermion fermion block with scalar products

$$
\begin{aligned}
& e_{p 2}^{(m-1) \dagger} u_{m}=\alpha_{p}^{(m)}, \\
& \left|\alpha_{p}^{(m)}\right|^{2}=\left(i s_{p 2}^{(m-1)}-i s_{p 2}^{(m)}\right) \\
& \frac{\prod_{q=m+1}^{k_{1}}\left(i s_{p 2}^{(m-1)}-s_{q 1}^{(m)}\right) \prod_{q=1, q \neq p}^{k_{2}}\left(i s_{p 2}^{(m-1)}-i s_{q 2}^{(m-1)}\right)}{\prod_{q=1, q \neq p}^{k_{2}}\left(i s_{p 2}^{(m-1)}-i s_{q 2}^{(m)}\right) \prod_{q=m}^{k_{1}}\left(i s_{p 2}^{(m-1)}-s_{q 1}^{(m-1)}\right)}, \\
& p=1, \ldots, k_{2} \text {, }
\end{aligned}
$$

where the moduli squared of the complex anticommuting Gelfand-Tzetlin coordinates $\xi_{p}^{(m)}$ are given by

$$
\left|\xi_{p}^{(m)}\right|^{2}=i s_{p 2}^{(m)}-i s_{p 2}^{(m-1)}, \quad p=1, \ldots, k_{2}
$$

As at the first level, $b_{p}^{(m)}$ can be commuting or anticommuting, and we write $w_{p}^{(m)}=$ $b_{p}^{(m)}, p=m+1, \ldots, k_{1}$ for the commuting and $\beta_{p}^{(m)}=b_{k_{1}+p}^{(m)}, p=1, \ldots, k_{2}$ for the anticommuting ones, 


$$
\begin{aligned}
\left|w_{p}^{(m)}\right|^{2}= & \frac{\prod_{q=m}^{k_{1}}\left(s_{p 1}^{(m)}-s_{q 1}^{(m-1)}\right) \prod_{q=1}^{k_{2}}\left(s_{p 1}^{(m)}-i s_{q 2}^{(m)}\right)}{\prod_{q=1}^{k_{2}}\left(s_{p 1}^{(m)}-i s_{q 2}^{(m-1)}\right) \prod_{q=m+1, q \neq p}^{k_{1}}\left(s_{p 1}^{(m)}-s_{q 1}^{(m)}\right)}, p=m+1, \ldots, k_{1}, \\
\left|\beta_{p}^{(m)}\right|^{2}= & \left(\begin{array}{c}
\left.i s_{p 2}^{(m)}-i s_{p 2}^{(m-1)}\right) \\
\\
\quad \frac{\prod_{q=m}^{k_{1}}\left(i s_{p 2}^{(m)}-s_{q 1}^{(m-1)}\right) \prod_{q=1, q \neq p}^{k_{2}}\left(i s_{p 2}^{(m)}-i s_{q 2}^{(m)}\right)}{\prod_{q=1, q \neq p}^{k_{2}}\left(i s_{p 2}^{(m)}-i s_{q 2}^{(m-1)}\right) \prod_{q=m+1}^{k_{1}}\left(i s_{p 2}^{(m)}-s_{q 1}^{(m)}\right)} \\
p=1, \ldots, k_{2}
\end{array} .\right.
\end{aligned}
$$

The invariant measures at these $k_{1}$ levels are given by

$$
\begin{gathered}
d \mu\left(u_{m}\right)=2^{k_{2}-k_{1}+m} \frac{B_{\left(k_{1}-m\right) k_{2}}\left(s^{(m)}\right)}{B_{\left(k_{1}-m+1\right) k_{2}}\left(s^{(m-1)}\right)} d\left[s_{1}^{(m)}\right] d\left[\vartheta^{(m)}\right] d\left[\xi^{(m)}\right], \\
m=1, \ldots, k_{1},
\end{gathered}
$$

where we use the notation

$$
d\left[s_{1}^{(m)}\right]=\prod_{p=m+1}^{k_{1}} d s_{p 1}^{(m)}, \quad d\left[\vartheta^{(m)}\right]=\prod_{p=m}^{k_{1}} d \vartheta_{p}^{(m)} \quad \text { and } \quad d\left[\xi^{(m)}\right]=\prod_{p=1}^{k_{2}} d \xi_{p}^{(m) *} d \xi_{p}^{(m)}
$$

for the products of the differentials. For the domain of integration of the commuting variables we find

$$
\begin{gathered}
s_{(p-1) 1}^{(m-1)} \leq s_{p 1}^{(m)} \leq s_{p 1}^{(m-1)}, \quad p=m+1, \ldots, k_{1} \\
0 \leq \vartheta_{p}^{(m)}<2 \pi, \quad p=m, \ldots, k_{1}
\end{gathered}
$$

where $m=1, \ldots, k_{1}$. Again, the Berezin integrals are evaluated as usual.

The $k_{1}^{\text {th }}$ level is the last one where anticommuting variables appear explicitly, the further $k_{2}$ steps are exactly like in the original Gelfand-Tzetlin problem. For the sake of completeness, we give the formulae in the following. At the level $m=$ $k_{1}+1, \ldots, k_{1}+k_{2}$, the Gelfand-Tzetlin equation has $k_{1}+k_{2}-m$ eigenvalues $i s_{p 2}^{(m)}=$ $s_{k_{1}+p}^{(m)}, p=m-k_{1}+1, \ldots, k_{2}$ which do not contain further anticommuting GelfandTzetlin coordinates and $k_{1}+k_{2}-m$ eigenvectors $e_{p 2}^{(m)}=e_{k_{1}+p}^{(m)}, p=m-k_{1}+1, \ldots, k_{2}$. Since the order of the commuting and anticommuting variables is now interchanged in the eigenvectors and the vectors $u_{m}$, we write the scalar products in the form

$$
\begin{aligned}
e_{p 2}^{(m-1) \dagger} u_{m} & =\sqrt{\left|v_{p}^{(m)}\right|^{2}} \exp \left(i \vartheta_{p}^{(m)}\right) \\
\left|v_{p}^{(m)}\right|^{2} & =\frac{\prod_{q=m+1}^{k_{2}}\left(i s_{p 2}^{(m-1)}-i s_{q 2}^{(m)}\right)}{\prod_{q=m, q \neq p}^{k_{2}}\left(i s_{p 2}^{(m-1)}-i s_{q 2}^{(m-1)}\right)}, \quad p=m-k_{1}, \ldots, k_{2}
\end{aligned}
$$

Similarly, we write the expansion coefficients at the levels $m=k_{1}+1, \ldots, k_{1}+k_{2}$ in the form $w_{p}^{(m)}=b_{p}^{(m)}, p=m+1, \ldots, k_{1}+k_{2}$ and obtain for the the moduli squared 


$$
\left|w_{p}^{(m)}\right|^{2}=\frac{\prod_{q=m}^{k_{2}}\left(i s_{p 2}^{(m)}-i s_{q 2}^{(m-1)}\right)}{\prod_{q=m+1, q \neq p}^{k_{2}}\left(i s_{p 2}^{(m)}-i s_{q 2}^{(m)}\right)}, \quad p=m-k_{1}+1, \ldots, k_{1}+k_{2}
$$

The invariant measures at these $k_{2}$ levels are given by

$$
\begin{array}{r}
d \mu\left(u_{m}\right)=2^{m-k_{1}-k_{2}} \frac{\Delta_{k_{1}+k_{2}-m}\left(i s_{2}^{(m)}\right)}{\Delta_{k_{1}+k_{2}-m+1}\left(i s_{2}^{(m-1)}\right)} d\left[s_{2}^{(m)}\right] d\left[\vartheta^{(m)}\right], \\
m=k_{1}+1, \ldots, k_{1}+k_{2}
\end{array}
$$

where we use the notation

$$
d\left[s_{2}^{(m)}\right]=\prod_{p=m-k_{1}+1}^{k_{2}} d s_{p 2}^{(m)} \quad \text { and } \quad d\left[\vartheta^{(m)}\right]=\prod_{p=m-k_{1}}^{k_{2}} d \vartheta_{p}^{(m)}
$$

for the products of the differentials. The domain of integration of the commuting variables is given by

$$
\begin{gathered}
i s_{(p-1) 2}^{(m-1)} \leq i s_{p 2}^{(m)} \leq i s_{p 2}^{(m-1)}, \quad p=m-k_{1}+1, \ldots, k_{2} \\
0 \leq \vartheta_{p}^{(m)}<2 \pi, \quad p=m-k_{1}, \ldots, k_{2}
\end{gathered}
$$

where $m=k_{1}+1, \ldots, k_{1}+k_{2}$.

2.8. Matrix Elements and Supergroup Measure. Using the recursion, we can now express all elements of the unitary supermatrix in Gelfand-Tzetlin coordinates. From Eqs. (2.39),(2.43) and (2.44) we find

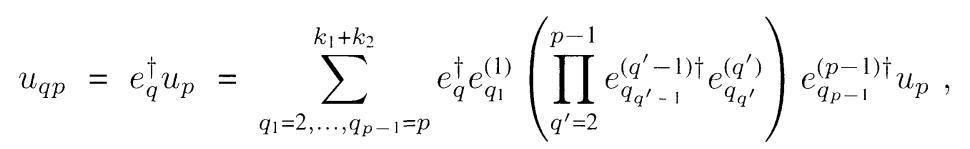

where all scalar products are given explicitly in the last subsection. The invariant Haar measure of the unitary supergroup is the product of the invariant measures evaluated above,

$$
d \mu(u)=\frac{2^{-\left(k_{1}+k_{2}\right)\left(k_{1}+k_{2}-1\right) / 2}}{B_{k_{1} k_{2}}(s)} \prod_{m=1}^{k_{1}} d\left[s_{1}^{(m)}\right] d\left[\vartheta^{(m)}\right] d\left[\xi^{(m)}\right] \prod_{m=k_{1}+1}^{k_{1}+k_{2}} d\left[s_{2}^{(m)}\right] d\left[\vartheta^{(m)}\right]
$$

which does not depend on the Gelfand-Tzetlin eigenvalues that parametrize the unitary supergroup. This yields inserted in Eq. (2.5) an explicit expression for the volume element of the Hermitian supermatrices.

Finally, we write the elements of the Hermitian supermatrix $\sigma$ defined in Eq. (2.1),

$$
\sigma_{q p}=u_{q}^{\dagger} s u_{p}
$$

as functions of the Gelfand-Tzetlin eigenvalues. Without loss of generality we assume $q \geq p$ and obtain 


$$
\sigma_{q p}=u_{q}^{\dagger} s^{(p-1)} u_{p}=\sum_{q^{\prime}=p}^{k_{1}+k_{2}} s_{q^{\prime}}^{(p-1)} u_{q}^{\dagger} e_{q^{\prime}}^{(p-1)} e_{q^{\prime}}^{(p-1) \dagger} u_{p}
$$

where all scalar products are given by Eq. (2.59). For the matrix elements on the diagonal we find

$$
\sigma_{p p}=\operatorname{trg} s^{(p-1)}-\operatorname{trg} s^{(p)},
$$

where we use the notation $s^{\left(k_{1}+k_{2}\right)}=0$. This remarkable result is a generalization of the corresponding formula for ordinary Hermitian matrices [17]. The derivation of the expression (2.63) requires some lengthy calculations. Again, a more elegant proof involving representation theory is likely to exist.

\section{Harish-Chandra-Itzykson-Zuber Integral}

As shown by Shatashvili [17], the Gelfand-Tzetlin method is, due to its recursive structure, very well suited for the study of correlation functions, i.e. angular averages, in the Itzykson-Zuber model $[15,14]$. We will demonstrate in the following that the same is true in the supersymmetric case. The supersymmetric version of the HarishChandra-Itzykson-Zuber integral [9]

$$
I(s, r)=\int \exp \left(i \operatorname{trg} u^{-1} s u r\right) d \mu(u)
$$

where $r$ is a diagonal matrix of the type (2.3) was first evaluated by generalizing the diffusion equation technique of Itzykson and Zuber [15] to supermatrices. We restrict ourselves to the rederivation of the integral (3.1), the generalization of Shatashvili's correlation functions is then straightforward.

With Eqs. (2.2) and (2.63) we obtain

$$
\begin{aligned}
\operatorname{trg} u^{-1} \text { sur } & =\sum_{p=1}^{k_{1}} \sigma_{p p} r_{p}-\sum_{p=1}^{k_{2}} \sigma_{\left(k_{1}+p\right)\left(k_{1}+p\right)} r_{k_{1}+p} \\
& =\sum_{p=1}^{k_{1}}\left(\operatorname{trg} s^{(p-1)}-\operatorname{trg} s^{(p)}\right) r_{p 1} \\
& -\sum_{p=1}^{k_{2}}\left(\operatorname{trg} s^{\left(k_{1}+p-1\right)}-\operatorname{trg} s^{\left(k_{1}+p\right)}\right) i r_{p 2}
\end{aligned}
$$

where the traces are given by

$$
\begin{aligned}
\operatorname{trg} s^{(p)} & =\sum_{q=p+1}^{k_{1}} s_{q 1}^{(p)}-\sum_{q=1}^{k_{2}} i s_{q 2}^{(p)}, \quad p=0, \ldots, k_{1}, \\
\operatorname{trg} s^{\left(k_{1}+p\right)} & =-\sum_{q=p+1}^{k_{2}} i s_{q 2}^{\left(k_{1}+p\right)}, \quad p=0, \ldots, k_{2} .
\end{aligned}
$$

The structure of the invariant measure (2.60) and the second of Eqs. (3.3) show that the integrals over the variables $s^{\left(k_{1}+p\right)}, \vartheta^{\left(k_{1}+p\right)}, p=1, \ldots, k_{2}$ amount to nothing else but 
the ordinary Harish-Chandra-Itzykson-Zuber integral over the group $U\left(k_{2}\right)$, exactly as calculated by Shatashvili. The remaining expression

$$
\begin{gathered}
I(s, r)=\frac{i^{k_{2}\left(k_{2}-1\right) / 2}(2 \pi)^{k_{2}\left(k_{2}+1\right) / 2}}{2^{\left(k_{1}+k_{2}\right)\left(k_{1}+k_{2}-1\right) / 2}} \frac{1}{B_{k_{1} k_{2}}(s) \Delta_{k_{2}}\left(i r_{2}\right)} \\
\int \prod_{p=1}^{k_{1}} d\left[s_{1}^{(p)}\right] d\left[\vartheta^{(p)}\right] d\left[\xi^{(p)}\right] \exp \left(i\left(\operatorname{trg} s^{(p-1)}-\operatorname{trg} s^{(p)}\right) r_{p 1}\right) \\
\operatorname{det}\left[\exp \left(-i\left(i s_{q 2}^{\left(k_{1}\right)} i r_{q^{\prime} 2}\right)\right)\right]_{q, q^{\prime}=1, \ldots, k_{2}}
\end{gathered}
$$

allows an easy evaluation of the integrals over the anticommuting variables. From Eq. (2.49) we find

$$
\begin{aligned}
\operatorname{trg} s^{(p-1)}-\operatorname{trg} s^{(p)} & =\operatorname{tr} s_{1}^{(p-1)}-\operatorname{tr} s_{1}^{(p)}+\sum_{q=1}^{k_{2}}\left|\xi_{q}^{(p)}\right|^{2} \\
i s_{q 2}^{\left(k_{1}\right)} & =i s_{q 2}+\sum_{p=1}^{k_{1}}\left|\xi_{q}^{(p)}\right|^{2} .
\end{aligned}
$$

The determinant in Eq. (3.4) is written in the Laplace expansion, yielding as the first term $\exp \left(-i \operatorname{tr} i s_{2}^{\left(k_{1}\right)} i r_{2}\right)$. Collecting all contributions with anticommuting variables, we arrive at

$$
\begin{gathered}
\int \prod_{p=1}^{k_{1}} d\left[\xi^{(p)}\right] \exp \left(i \sum_{q=1}^{k_{2}}\left|\xi_{q}^{(p)}\right|^{2} r_{p 1}\right) \exp \left(-i \sum_{q=1}^{k_{2}}\left|\xi_{q}^{(p)}\right|^{2} i r_{q 2}\right) \\
=\prod_{p, q} \int d \xi_{q}^{(p) *} d \xi_{q}^{(p)} \exp \left(i\left(r_{p 1}-i r_{q 2}\right) \xi_{q}^{(p) *} \xi_{q}^{(p)}\right) \\
=\frac{1}{(i 2 \pi)^{k_{1} k_{2}}} \prod_{p, q}\left(r_{p 1}-i r_{q 2}\right)
\end{gathered}
$$

which is invariant under permutations and therefore also the result for all other terms in the Laplace expansion. The thus obtained expression

$$
\begin{aligned}
I(s, r)= & \frac{i^{k_{2}\left(k_{2}-1\right) / 2}(2 \pi)^{k_{2}\left(k_{2}+1\right) / 2}}{2^{\left(k_{1}+k_{2}\right)\left(k_{1}+k_{2}-1\right) / 2}(i 2 \pi)^{k_{1} k_{2}}} \\
& \frac{\prod_{p, q}\left(r_{p 1}-i r_{q 2}\right)}{B_{k_{1} k_{2}}(s) \Delta_{k_{2}}\left(i r_{2}\right)} \operatorname{det}\left[\exp \left(i s_{p 2} r_{q 2}\right)\right]_{p, q=1, \ldots, k_{2}} \\
& \int \prod_{p=1}^{k_{1}} d\left[s_{1}^{(p)}\right] d\left[\vartheta^{(p)}\right] \exp \left(i\left(\operatorname{tr} s_{1}^{(p-1)}-\operatorname{tr} s_{1}^{(p)}\right) r_{p 1}\right)
\end{aligned}
$$

contains again nothing else but the ordinary Harish-Chandra-Itzykson-Zuber integral as evaluated in reference [17], but this time over the group $U\left(k_{1}\right)$. The final result 


$$
\begin{aligned}
& I(s, r)=\frac{i^{\left(\left(k_{1}-k_{2}\right)^{2}-\left(k_{1}+k_{2}\right)\right) / 2}(2 \pi)^{\left(\left(k_{1}-k_{2}\right)^{2}+\left(k_{1}+k_{2}\right)\right) / 2}}{2^{\left(k_{1}+k_{2}\right)\left(k_{1}+k_{2}-1\right) / 2}} \\
& \frac{\left.\left.\operatorname{det}\left[\exp \left(i s_{p 1} r_{q 1}\right)\right)\right]_{p, q=1, \ldots, k_{1}} \operatorname{det}\left[\exp \left(i s_{p 2} r_{q 2}\right)\right)\right]_{p, q=1, \ldots, k_{2}}}{B_{k_{1} k_{2}}(s) B_{k_{1} k_{2}}(r)}
\end{aligned}
$$

is a generalization of the formula derived in reference [9] which is found from Eq. (3.8) in the case $k_{1}=k_{2}$.

The calculation performed here shows some kind of a coset structure. The integrals over the subgroups $U\left(k_{1}\right)$ and $U\left(k_{2}\right)$ have the well known ordinary form and are completely decoupled. Moreover, the integrals over the anticommuting variables are also decoupled and explore the coset manifold $U\left(k_{1} / k_{2}\right) /\left(U\left(k_{1}\right) \otimes U\left(k_{2}\right)\right)$. These features will become more transparent in the following section.

\section{Aspects of Representation Theory}

In the representation theory of the ordinary unitary group $U(k)$, Gelfand [6] introduced the irreducible basis corresponding to the group chain

$$
U(k) \supset U(k-1) \supset \cdots \supset U(2) \supset U(1),
$$

which is determined by $k(k+1) / 2$ positive integers $x_{\jmath}^{(i)}, i=0, \ldots, k-1, j=i+1, \ldots, k$ satisfying the condition

$$
x_{j-1}^{(\imath-1)} \leq x_{\jmath}^{(\imath)} \leq x_{\jmath}^{(\imath-1)} .
$$

The basis vectors are eigenfunctions of the complete set of commuting operators in the group chain (4.1) and are labeled by the Gelfand pattern

$$
\begin{array}{cccccccc}
x_{1}^{(0)} & & x_{2}^{(0)} & & x_{3}^{(0)} & \cdots & & x_{k}^{(0)} \\
& x_{2}^{(1)} & & x_{3}^{(1)} & \cdots & & x_{k}^{(1)} & \\
& & \vdots & & & \\
& & x_{k-1}^{(k-2)} & & x_{k}^{(k-2)} & & & \\
& & & x_{k}^{(k-1)} & & &
\end{array}
$$

which is related to the Young and Weyl tableaux. The $j^{\text {th }}$ row in this pattern belongs to the irreducible representation of the group $U(k+1-j)$. Excellent reviews on these topics can be found in references [4] and [2]. The Gelfand-Tzetlin method [8, 7] shows a close relationship between these irreducible representations and an explicit coordinate system on the parameter space of the unitary group $U(k)$.

In view of this discussion, we are led to the conclusion that the construction of coordinates on the parameter space of the supergroup $U\left(k_{1} / k_{2}\right)$ performed in Sect. 2 has implications for the representation theory. It was shown in references [10] and [11] that the group $U(1 / 1)$ can be represented, analogously to the ordinary group $S U(2)$, by graded Wigner functions. These representation functions are matrix elements in a space which is formally similar to a Hilbert space. Anticommuting variables play a role formally similar to the angular momentum quantum numbers and appear also in the orthonormality relation. Hence, there exists a representation of the supergroup $U(1 / 1)$ determined and labeled by a complex anticommuting variable. These considerations can now be generalized to the unitary supergroup $U\left(k_{1} / k_{2}\right)$. We view the commuting 
Gelfand-Tzetlin eigenvalues $s_{p 1}^{(m)}, m=0, \ldots, k_{1}-1, p=m+1, \ldots, k_{1}$ and $i s_{p 2}^{(m)}, m=$ $k_{1}, \ldots, k_{1}+k_{2}-1, p=m-k_{1}+1, \ldots, k_{2}$ as positive integers subject to the conditions

$$
\begin{gathered}
s_{(p-1) 1}^{(m-1)} \leq s_{p 1}^{(m)} \leq s_{p 1}^{(m-1)}, \\
i s_{(p-1) 2}^{(m-1)} \leq i s_{p 2}^{(m)} \leq i s_{p 2}^{(m-1)},
\end{gathered}
$$

which were already stated for the continuous variables in Eqs. (2.53) and (2.58). Naturally, there is no interpretation of this sort for the anticommuting variables $\xi_{p}^{(m)}, \xi_{p}^{(m) *}, m=1, \ldots, k_{1}, p=1, \ldots, k_{2}$. We obtain the generalized Gelfand pattern

$$
\begin{aligned}
& \begin{array}{llllllll}
s_{11} & & s_{21} & & s_{31} & \cdots & & s_{k_{1} 1} \\
& s_{21}^{(1)} & & s_{31}^{(1)} & \cdots & & s_{k_{1} 1}^{(1)} &
\end{array} \\
& \begin{array}{lcl}
s_{\left(k_{1}-1\right) 1}^{\left(k_{1}-2\right)} & s_{k_{1} 1}^{\left(k_{1}-2\right)} \\
& s_{k_{1} 1}^{\left(k_{1}-1\right)}
\end{array} \\
& \left|\xi_{1}^{(1)}\right|^{2} \quad\left|\xi_{2}^{(1)}\right|^{2} \quad \ldots \quad \quad\left|\xi_{k_{2}}^{(1)}\right|^{2} \\
& \left|\xi_{1}^{(2)}\right|^{2} \quad\left|\xi_{2}^{(2)}\right|^{2} \quad \cdots \quad \quad\left|\xi_{k_{2}}^{(2)}\right|^{2} \\
& \begin{array}{cccc}
\vdots & & \vdots \\
\left.\xi_{1}^{\left(\dot{k}_{1}\right)}\right|^{2} & \left|\xi_{2}^{\left(k_{1}\right)}\right|^{2} & \cdots & \left|\xi_{k_{2}}^{\left(\bar{k}_{1}\right)}\right|^{2}
\end{array}
\end{aligned}
$$

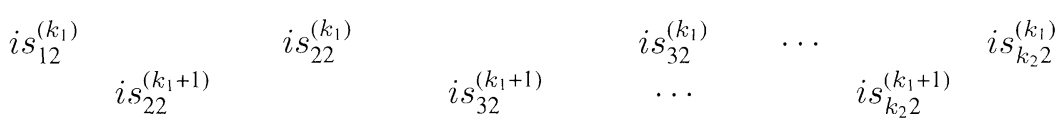

$$
\begin{aligned}
& i s_{\left(k_{2}-1\right) 2}^{\left(k_{1}+k_{2}-2\right)} \quad \begin{array}{l}
i s_{k_{2} 2}^{\left(k_{1}+k_{2}-2\right)} \\
i s_{k_{2} 2}^{\left(k_{1}+k_{2}-1\right)}
\end{array}
\end{aligned}
$$

which labels representations of the unitary supergroup $U\left(k_{1} / k_{2}\right)$. The generalized Gelfand pattern consists of two triangular subpatterns for the commuting and one rectangular subpattern for the anticommuting variables. Obviously, the two triangular subpatterns label irreducible bases of the ordinary unitary groups $U\left(k_{1}\right)$ and $U\left(k_{2}\right)$ and hence both together label irreducible bases for the direct product $U\left(k_{1}\right) \otimes U\left(k_{2}\right)$ which is a subgroup of the supergroup $U\left(k_{1} / k_{2}\right)$. The remaining coset $U\left(k_{1} / k_{2}\right) /\left(U\left(k_{1}\right) \otimes\right.$ $\left.U\left(k_{2}\right)\right)$ is represented by the rectangular pattern of anticommuting variables. From the construction, we may conclude that the generalized Gelfand pattern labels the basis which corresponds to the supergroup chain

$$
U\left(k_{1} / k_{2}\right) \supset U\left(k_{1}-1 / k_{2}\right) \supset \cdots \supset U\left(1 / k_{2}\right) \supset U\left(k_{2}\right) \supset \cdots \supset U(2) \supset U(1)
$$

and that the basis functions are eigenfunctions of the complete set of commuting operators in this chain. The structure of the generalized Gelfand pattern is also reflected in the harmonic analysis to be discussed in a forthcoming publication.

There is already a theory [16] of finite-dimensional representations of the superalgebras $g l\left(k_{1} / k_{2}\right)$ and $u\left(k_{1} / k_{2}\right)$ involving a Gelfand pattern. Although anticommuting variables do not appear in those, there ought to be a connection to the generalized Gelfand pattern (4.5) for the supergroup $U\left(k_{1} / k_{2}\right)$ which contains anticommuting variables explicitly. Moreover, Balantekin and Bars [1] constructed representations of 
the unitary supergroup in terms of extended Young supertableaux. Again, anticommuting variables do not appear explicitly in those tableaux and it remains an open problem to find the relation to the generalized Gelfand pattern (4.5).

\section{Summary and Discussion}

We generalized the Gelfand-Tzetlin method to unitary supergroups $U\left(k_{1} / k_{2}\right)$ through a purely algebraic construction. By slicing off the first column of the unitary supermatrix, a projection to a subspace is defined. The resulting generalized Gelfand-Tzetlin equations for the Gelfand-Tzetlin eigenvalues contain certain singularities which are a direct consequence of the presence of anticommuting variables. The moduli squared of the vector elements are expressed in differences of the eigenvalues of a Hermitian supermatrix and the Gelfand-Tzetlin eigenvalues. The solutions led to the definition of complex anticommuting Gelfand-Tzetlin coordinates. The structure of the solution suggests that a more sophisticated derivation than the direct calculation performed here must exist which should have a direct relationship to the representation theory. Upon evaluating the eigenvectors, a dual vector was found which defines a basis transformation analogous to the ordinary case. The invariant length element was calculated in the new variables. Remarkably, the corresponding metric tensor is diagonal which shows once more how symmetry adopted the Gelfand-Tzetlin eigenvalues are. The resulting Berezinian is the square root of the ratio of the two Berezinians for the spaces involved, again indicating a deeper meaning from the viewpoint of representation theory. The recursion of the method was performed to all levels. All elements of the unitary and the Hermitian supermatrices were expressed in the new variables. The total invariant measure of the unitary supergroup does not contain those new variables and is a simple product of all independent differentials. All of our findings reproduce the original Gelfand-Tzetlin results for $k_{1}=0$ or $k_{2}=0$. The calculation of the supersymmetric Harish-Chandra-Itykson-Zuber integral exhibits the inherent coset structure of the generalized Gelfand-Tzetlin coordinates.

Taking into consideration a previously derived representation of the supergroup $U(1 / 1)$, we concluded that the generalized Gelfand-Tzetlin method has a close relationship to the representation theory of supergroups. A generalized Gelfand pattern was constructed which labels the basis of eigenfunctions of a complete set of commuting operators in the supergroup chain which generalizes the group chain discussed by Gelfand. The pattern contains two triangular subpatterns determining representations of the maximal, ordinary product group which is a subgroup of $U\left(k_{1} / k_{2}\right)$. The remaining pattern of anticommuting labels represents the coset. Once more, the author would like to emphasize that a deeper understanding of those representations is highly desirable, also from the viewpoint of applications. The harmonic analysis on the spaces discussed here will be published elsewhere.

Acknowledgement. I am grateful to J. Lyng Petersen and H. Plesner Jakobsen for stimulating discussions. Special thanks to V. Putkaradze for helping me to read the Russian references. I acknowledge financial support from the Max Planck Gesellschaft as an Otto Hahn fellow and from the Danish Research Council. 


\section{A. Appendix: Derivation of the Moduli Squared for $k_{1}=k_{2}=2$}

In the case $k_{1}=k_{2}=2$ there are three Gelfand-Tzetlin eigenvalues and four Eqs. (2.13). From the first two equations, which do not involve $z_{1}, z_{2}$, we find

$$
\left|v_{1}^{(1)}\right|^{2}=\frac{s_{11}-s_{21}^{(1)}}{s_{11}-s_{21}}\left(1-\frac{i s_{12}-s_{21}}{i s_{12}-s_{21}^{(1)}}\left|\alpha_{1}^{(1)}\right|^{2}-\frac{i s_{22}-s_{21}}{i s_{22}-s_{21}^{(1)}}\left|\alpha_{2}^{(1)}\right|^{2}\right)
$$

and analogously for $\left|v_{2}^{(1)}\right|^{2}$ by exchanging $s_{11}$ and $s_{21}$. After inserting this into the last two equations, inverting them and taking the limits $z_{1}, z_{2} \rightarrow \infty$ we find

$$
\begin{gathered}
0=\left(i s_{12}-i s_{12}^{(1)}\right)\left(i s_{22}-i s_{12}^{(1)}\right)+\frac{\left(i s_{12}-s_{11}\right)\left(i s_{12}-s_{21}\right)\left(i s_{22}-i s_{12}^{(1)}\right)}{i s_{12}-s_{21}^{(1)}}\left|\alpha_{1}^{(1)}\right|^{2} \\
+\frac{\left(i s_{22}-s_{11}\right)\left(i s_{22}-s_{21}\right)\left(i s_{12}-i s_{12}^{(1)}\right)}{i s_{22}-s_{21}^{(1)}}\left|\alpha_{2}^{(1)}\right|^{2} \\
+2 \frac{\left(i s_{12}-s_{11}\right)\left(i s_{12}-s_{21}\right)\left(i s_{22}-s_{11}\right)\left(i s_{22}-s_{21}\right)}{\left(i s_{12}-s_{21}^{(1)}\right)\left(i s_{22}-s_{21}^{(1)}\right)}\left|\alpha_{1}^{(1)}\right|^{2}\left|\alpha_{2}^{(1)}\right|^{2}
\end{gathered}
$$

and similarly for $i s_{22}^{(1)}$. The most general form of the Gelfand-Tzetlin eigenvalues is

$$
i s_{p 2}^{(1)}=c_{p 0}+c_{p 11}\left|\alpha_{1}^{(1)}\right|^{2}+c_{p 12}\left|\alpha_{2}^{(1)}\right|^{2}+c_{p 2}\left|\alpha_{1}^{(1)}\right|^{2}\left|\alpha_{2}^{(1)}\right|^{2}, \quad p=1,2 .
$$

This ansatz yields two sets of solutions for the coefficients $c_{p 0}, c_{p 11}, c_{p 12}$ and $c_{p 2}$. We arrive at

$$
\begin{aligned}
i s_{12}^{(1)}=i s_{12}+\frac{\left(i s_{12}-\right.}{\left.s_{11}\right)\left(i s_{12}-s_{21}\right)} & \left.\alpha_{1}^{(1)}\right|^{2} \\
& \left(1+\frac{\left(i s_{22}-s_{11}\right)\left(i s_{22}-s_{21}\right)}{\left(i s_{22}-i s_{12}\right)\left(i s_{22}-s_{21}^{(1)}\right)}\left|\alpha_{2}^{(1)}\right|^{2}\right)
\end{aligned}
$$

and similarly for $i s_{22}^{(1)}$. Again, the non-trivial part of the Gelfand-Tzetlin eigenvalues in the fermion fermion block is the modulus squared of anticommuting variables. We introduce the anticommuting Gelfand-Tzetlin coordinates $\xi_{p}^{(1)}, p=1,2$ where

$$
\left|\xi_{p}^{(1)}\right|^{2}=i s_{p 2}^{(1)}-i s_{p 2}
$$

Collecting everything and solving for the modulus squared of the vector elements yields

$$
\begin{aligned}
\left|v_{1}^{(1)}\right|^{2} & =\frac{\left(s_{11}-s_{21}^{(1)}\right)\left(s_{11}-i s_{12}\right)\left(s_{11}-i s_{22}\right)}{\left(s_{11}-s_{21}\right)\left(s_{11}-i s_{12}^{(1)}\right)\left(s_{11}-i s_{22}^{(1)}\right)}, \\
\left|v_{2}^{(1)}\right|^{2} & =\frac{\left(s_{21}-s_{21}^{(1)}\right)\left(s_{21}-i s_{12}\right)\left(s_{21}-i s_{22}\right)}{\left(s_{21}-s_{11}\right)\left(s_{21}-i s_{12}^{(1)}\right)\left(s_{21}-i s_{22}^{(1)}\right)},
\end{aligned}
$$




$$
\begin{aligned}
\left|\alpha_{1}^{(1)}\right|^{2} & =\frac{\left(i s_{12}-i s_{12}^{(1)}\right)\left(i s_{12}-s_{21}^{(1)}\right)\left(i s_{12}-i s_{22}\right)}{\left(i s_{12}-i s_{22}^{(1)}\right)\left(i s_{12}-s_{11}\right)\left(i s_{12}-s_{21}\right)}, \\
\left|\alpha_{2}^{(1)}\right|^{2} & =\frac{\left(i s_{22}-i s_{22}^{(1)}\right)\left(i s_{22}-s_{21}^{(1)}\right)\left(i s_{22}-i s_{12}\right)}{\left(i s_{22}-i s_{12}^{(1)}\right)\left(i s_{22}-s_{11}\right)\left(i s_{22}-s_{21}\right)} .
\end{aligned}
$$

These calculations are not completely trivial. Properties of the anticommuting variables and rules for manipulations with Vandermonde determinants have to be used.

\section{B. Appendix: General Derivation of the Moduli Squared}

The first $k_{1}$ of Eqs. (2.13) can be viewed as a system of linear equations for the moduli squared of the commuting matrix elements. Define a $k_{1}$ component vector $\widetilde{v}$ with entries $\left|v_{p}^{(1)}\right|^{2}$, a $k_{1} \times k_{1}$ matrix $M$ with entries

$$
\begin{aligned}
& M_{1 q}=1, \quad q=1, \ldots, k_{1} \\
& M_{p q}=\frac{1}{s_{q 1}-s_{p 1}^{(1)}}, \quad p=2, \ldots, k_{1}, q=1, \ldots, k_{1},
\end{aligned}
$$

and a $k_{1}$ component vector $a$ with entries

$$
\begin{aligned}
& a_{1}=1-\sum_{q=1}^{k_{2}}\left|\alpha_{q}\right|^{2}, \\
& a_{p}=-\sum_{q=1}^{k_{2}} \frac{\left|\alpha_{q}\right|^{2}}{i s_{q 2}-s_{p 1}^{(1)}}, \quad p=2, \ldots, k_{1} .
\end{aligned}
$$

The resulting linear system

$$
M \widetilde{v}=a
$$

can now be solved with Cramer's rule. The $k_{1} \times k_{1}$ matrix $M^{(p)}$ is found from $M$ by replacing the $p^{\text {th }}$ column with $a$, thus we have

$$
\left|v_{p}^{(1)}\right|^{2}=\widetilde{v}_{p}=\frac{\operatorname{det} M^{(p)}}{\operatorname{det} M}
$$

Expanding $\operatorname{det} M^{(p)}$ and regrouping terms yields

$$
\left|v_{p}^{(1)}\right|^{2}=(-1)^{p+1} \frac{\operatorname{det} \widetilde{M}^{(p)}}{\operatorname{det} M}+\sum_{q=1}^{k_{2}} \frac{\operatorname{det} M^{(p, q)}}{\operatorname{det} M}\left|\alpha_{q}^{(1)}\right|^{2},
$$

where the $\left(k_{1}-1\right) \times\left(k_{1}-1\right)$ matrix $\widetilde{M}^{(p)}$ is constructed from $M$ by removing the first row and the $p^{\text {th }}$ column and the $k_{1} \times k_{1}$ matrix $M^{(p, q)}$ is found from $M$ by replacing the $p^{\text {th }}$ column with 1 in the first entry and with $1 /\left(i s_{q 2}-s_{p^{\prime} 1}^{(1)}\right)$ in the entries $p^{\prime}=2, \ldots, k_{1}$. To evaluate the determinants further, we make use of Cauchy's lemma, 


$$
\operatorname{det}\left[\frac{1}{x_{p}-y_{q}}\right]_{p, q=1, \ldots, k}=(-1)^{k(k-1) / 2} \frac{\Delta_{k}(x) \Delta_{k}(y)}{\prod_{p, q}\left(x_{p}-y_{q}\right)}
$$

for two sets of variables $x_{p}, y_{p}, p=1, \ldots, k$. Using properties of the Vandermonde determinant we arrive at

$$
\left|v_{p}^{(1)}\right|^{2}=\frac{\prod_{q=2}^{k_{1}}\left(s_{p 1}-s_{q 1}^{(1)}\right)}{\prod_{q=1, q \neq p}^{k_{1}}\left(s_{p 1}-s_{q 1}\right)}\left(1+\sum_{p^{\prime}=1}^{k_{2}} \frac{\prod_{q=1, q \neq p}^{k_{1}}\left(i s_{p^{\prime} 2}-s_{q 1}\right)}{\prod_{q=2}^{k_{1}}\left(i s_{p^{\prime} 2}-s_{q 1}^{(1)}\right)}\left|\alpha_{p^{\prime}}^{(1)}\right|^{2}\right)
$$

which after inserting into the last $k_{2}$ of Eqs. (2.13) and regrouping terms gives

$$
\frac{z_{p}}{i s_{p 2}^{(1)}}=\frac{\prod_{q=2}^{k_{1}}\left(i s_{p 2}^{(1)}-s_{q 1}^{(1)}\right)}{\prod_{q=1}^{k_{2}}\left(i s_{p 2}^{(1)}-i s_{q 2}\right)}\left(1-\sum_{p^{\prime}=1}^{k_{2}} \frac{\prod_{q=1}^{k_{1}}\left(i s_{p^{\prime} 2}-s_{q 1}\right)}{\left(i s_{p^{\prime} 2}-i s_{p 2}^{(1)}\right) \prod_{q=2}^{k_{1}}\left(i s_{p^{\prime} 2}-s_{q 1}^{(1)}\right)}\left|\alpha_{p^{\prime}}^{(1)}\right|^{2}\right)
$$

allowing to invert both sides of the equation and to take the limit $z_{p} \rightarrow \infty$. With numbers $\kappa_{p}, p=1, \ldots, k_{2}$ which take the values zero or one we find the system

$0=\sum_{\kappa=0}^{k_{2}} \kappa ! \sum_{\sum_{p^{\prime \prime}=1}^{k_{2}} \kappa_{p^{\prime \prime}}=\kappa} \prod_{p^{\prime}=1}^{k_{2}}\left(i s_{p^{\prime} 2}-i s_{p 2}^{(1)}\right)^{\delta_{0 \kappa_{p^{\prime}}}}\left(\frac{\prod_{q=1}^{k_{1}}\left(i s_{p^{\prime} 2}-s_{q 1}\right)}{\prod_{q=2}^{k_{1}}\left(i s_{p^{\prime} 2}-s_{q 1}^{(1)}\right)}\left|\alpha_{p^{\prime}}^{(1)}\right|^{2}\right)^{\delta_{1 \kappa_{p^{\prime}}}}$

of $k_{2}$ equations that can be solved with an ansatz of the type (A.3). It is of course easier to show that the expressions (2.20) satisfy Eqs. (B.7) and (B.9).

\section{Appendix: Invariant Length Element}

We write the modulus squared of the commuting vector elements and the anticommuting vector elements explicitly as functions of the anticommuting Gelfand-Tzetlin coordinates,

$$
\begin{aligned}
\left|v_{p}^{(1)}\right|^{2} & =\frac{\prod_{q=2}^{k_{1}}\left(s_{p 1}-s_{q 1}^{(1)}\right)}{\prod_{q=1, q \neq p}^{k_{1}}\left(s_{p 1}-s_{q 1}\right)} \prod_{q=1}^{k_{2}}\left(1+\frac{\left|\xi_{q}^{(1)}\right|^{2}}{s_{p 1}-i s_{q 2}}\right), \\
\alpha_{p}^{(1)} & =\xi_{p}^{(1)} \frac{\prod_{q=2}^{k_{1}} \sqrt{i s_{p 2}-s_{q 1}^{(1)}}}{\prod_{q=1, q \neq p}^{k_{1}} \sqrt{i s_{p 2}-s_{q 1}}} \prod_{q=1, q \neq p}^{k_{2}}\left(1+\frac{\left|\xi_{q}^{(1)}\right|^{2}}{2\left(i s_{p 2}-i s_{q 2}\right)}\right),
\end{aligned}
$$

and find for the differentials

$$
\begin{aligned}
& d\left|v_{p}^{(1)}\right|^{2}=\sum_{q=2}^{k_{1}} \frac{\left|v_{p}^{(1)}\right|^{2}}{s_{p 1}-s_{q 1}^{(1)}} d s_{q 1}^{(1)}+\sum_{q=1}^{k_{2}} \frac{\left|v_{p}^{(1)}\right|^{2}}{s_{p 1}-i s_{q 2}^{(1)}}\left(\xi_{q}^{(1)} d \xi_{q}^{(1) *}-\xi_{q}^{(1) *} d \xi_{q}^{(1)}\right) \\
& d \alpha_{p}^{(1)}=\sum_{q=2}^{k_{1}} \frac{\alpha_{p}^{(1)}}{2\left(i s_{p 2}-s_{q 1}^{(1)}\right)} d s_{q 1}^{(1)}+\frac{\alpha_{p}^{(1)}}{i s_{p 2}-i s_{p 2}^{(1)}} \xi_{p}^{(1) *} d \xi_{p}^{(1)}+ \\
& \sum_{q=1, q \neq p}^{k_{2}} \frac{\alpha_{p}^{(1)}}{2\left(i s_{p 2}-i s_{q 2}^{(1)}\right)}\left(\xi_{q}^{(1)} d \xi_{q}^{(1) *}-\xi_{q}^{(1) *} d \xi_{q}^{(1)}\right)
\end{aligned}
$$


which gives inserted into Eq. (2.29) for the invariant length element squared after a straightforward calculation

$$
\begin{aligned}
& d u_{1}^{\dagger} d u_{1}= \frac{1}{4} \sum_{p, q=2}^{k_{1}} u_{1}^{\dagger} \frac{1}{\left(s-s_{p 1}^{(1)}\right)\left(s-s_{q 1}^{(1)}\right)} u_{1} d s_{p 1}^{(1)} d s_{q 1}^{(1)}+ \\
& \frac{1}{4} \sum_{p, q=1}^{k_{2}} u_{1}^{\dagger} \frac{1}{\left(s-i s_{p 2}^{(1)}\right)\left(s-i s_{q 2}^{(1)}\right)} u_{1} \\
&\left.\sum_{p=1}^{k_{1}}\left|v_{p}^{(1)}\right|^{2}\left(d \vartheta_{p}^{(1)}\right)^{2}+\frac{1}{2} \sum_{p=1}^{k_{2}} \frac{\left|\alpha_{p}\right|^{2}}{i s_{p 2}-i s_{p 2}^{(1)}} d \xi_{p}^{(1) *}-\xi_{p}^{(1) *} d \xi_{p}^{(1)}\right)\left(\xi_{q}^{(1)} d \xi_{q}^{(1) *}-\xi_{q}^{(1) *} d \xi_{q}^{(1)}\right)+ \\
& \frac{1}{2} \sum_{p=2}^{k_{1}} \sum_{q=1}^{k_{2}} u_{1}^{\dagger} \frac{1}{\left(s-s_{p 1}^{(1)}\right)\left(s-i s_{q 2}^{(1)}\right)} u_{1} \\
& d s_{p 1}^{(1)}\left(\xi_{q}^{(1)} d \xi_{q}^{(1) *}-\xi_{q}^{(1) *} d \xi_{q}^{(1)}\right)
\end{aligned}
$$

This can be enormously simplified by observing that the bilinear forms in the above expression are related to the orthonormality relation of the eigenvectors. From Eq. (2.26) we find

$$
\begin{aligned}
\delta_{p q} & =e_{p}^{(1) \dagger} e_{q}^{(1)} \\
& =\left|b_{p}^{(1)}\right|^{2} u_{1}^{\dagger} \frac{1}{\left(s-s_{p}^{(1)}\right)\left(s-s_{q}^{(1)}\right)} u_{1}, \quad p, q=2, \ldots, k_{1}+k_{2}
\end{aligned}
$$

which yields

$$
\begin{aligned}
d u_{1}^{\dagger} d u_{1}=\frac{1}{4} \sum_{p=2}^{k_{1}} \frac{\left(d s_{p 1}^{(1)}\right)^{2}}{\left|w_{p}^{(1)}\right|^{2}}+\sum_{p=1}^{k_{1}}\left|v_{p}^{(1)}\right|^{2}\left(d \vartheta_{p}^{(1)}\right)^{2}+ \\
\frac{1}{2} \sum_{p=1}^{k_{2}}\left(\frac{i s_{p 2}-i s_{p 2}^{(1)}}{\left|\beta_{p}^{(1)}\right|^{2}}+\frac{\left|\alpha_{p}^{(1)}\right|^{2}}{i s_{p 2}-i s_{p 2}^{(1)}}\right) d \xi_{p}^{(1) *} d \xi_{p}^{(1)}
\end{aligned}
$$

where $\left|\alpha_{p}^{(1)}\right|^{2}$ and $\left|\beta_{p}^{(1)}\right|^{2}$ have to be inserted as they stand in formulae (2.20) and (2.25). A further simplification is obtained by expanding in $\left|\xi_{p}^{(1)}\right|^{2}$ and reordering terms,

$$
\begin{aligned}
\frac{i s_{p 2}-i s_{p 2}^{(1)}}{\left|\beta_{p}^{(1)}\right|^{2}}+\frac{\left|\alpha_{p}^{(1)}\right|^{2}}{i s_{p 2}-i s_{p 2}^{(1)}} & =\frac{\left|\alpha_{p}^{(1)}\right|^{2}}{i s_{p 2}-i s_{p 2}^{(1)}}\left(1+\frac{\left(i s_{p 2}-i s_{p 2}^{(1)}\right)^{2}}{\left|\alpha_{p}^{(1)}\right|^{2}\left|\beta_{p}^{(1)}\right|^{2}}\right) \\
& =\frac{\left|\alpha_{p}^{(1)}\right|^{2}}{i s_{p 2}-i s_{p 2}^{(1)}}\left(1+\left(1+\left|\xi_{p}^{(1)}\right|^{2} \gamma_{p}^{(1)}(s)\right)\right) \\
& =2 \frac{\left|\alpha_{p}^{(1)}\right|^{2}}{i s_{p 2}-i s_{p 2}^{(1)}} \sqrt{1+\left|\xi_{p}^{(1)}\right|^{2} \gamma_{p}^{(1)}(s)}
\end{aligned}
$$




$$
\begin{aligned}
& =2 \frac{\left|\alpha_{p}^{(1)}\right|^{2}}{i s_{p 2}-i s_{p 2}^{(1)}} \sqrt{\frac{\left(i s_{p 2}-i s_{p 2}^{(1)}\right)^{2}}{\left|\alpha_{p}^{(1)}\right|^{2}\left|\beta_{p}^{(1)}\right|^{2}}} \\
& =2 \sqrt{\frac{\left|\alpha_{p}^{(1)}\right|^{2}}{\left|\beta_{p}^{(1)}\right|^{2}}}
\end{aligned}
$$

where $\gamma_{p}^{(1)}(s)$ is defined through the expansion. Thus, we find the desired result (2.33).

\section{References}

1. Balantekin. A.B., Bars, I.: Dimension and Character Formulas for Lie Supergroups. J. Math. Phys. 22, 1149 (1981); Representations of Supergroups. J. Math. Phys. 22, 1810 (1981); Branching Rules for the Supergroups $S U(N / M)$ from those of $S U(N+M)$. J. Math. Phys. 23, 1239 (1982)

2. Barut, A.O., Raczka, R.: Theory of Group Representations and Applications. Warszawa: Polish Scientific Publishers, 1980

3. Berezin, F.A.: Introduction to Superanalysis, MPAM vol. 9. Dordrecht: D. Reidel Publishing, 1987

4. Chen, J.Q.: Group Representation Theory for Physicists. Singapore: World Scientific, 1989

5. Efetov, K.B.: Supersymmetry and Theory of Disordered Metals. Adv. Phys. 32, 53 (1983)

6. Gelfand, I.M.: The Center of an Infinitesimal Group Algebra (Russian). Mat. Sb. 26, 103 (1950)

7. Gelfand, I.M., Naimark, M.A.: Unitary Representations of Classical Groups (Russian). Trudi MIAN 36, 1 (1950)

8. Gelfand, I.M., Tzetlin, M.L.: Matrix Elements for the Unitary Groups (Russian). Dokl. Akad. Nauk. 71, 825 (1950)

9. Guhr, T.: Dyson's Correlation Functions and Graded Symmetry. J. Math. Phys. 32, 336 (1991)

10. Guhr, T.: Fourier-Bessel Analysis for Ordinary and Graded $2 \times 2$ Hermitean Matrices. J. Math. Phys. 34, 2523 (1993)

11. Guhr, T.: On the Graded Group U(1/1). J. Math. Phys. 34, 2541 (1993)

12. Guhr, T.: On the Level Density of Coupled Gaussian Unitary Ensembles. Nuc. Phys. A560, 223 (1993)

13. Guhr, T., Weidenmüller, H.A.: Isospin Mixing and Spectral Fluctuation Properties. Ann. Phys. (NY) 199, 412 (1990)

14. Harish-Chandra: Spherical Functions on a Semisimple Lie Group I. Am. J. Math. 80, 241 (1958)

15. Itzykson, C., Zuber, J.B.: The Planar Approximation II. J. Math. Phys. 21, 411 (1980)

16. Palev, T.D., Storlova, N.I., Van der Jeugt, J.: Finite-Dimensional Representations of the Quantum Superalgebra $U_{q}[g l(n / m)]$ and Related $q$-Identities. Commun. Math. Phys. 166, 367 (1994) and references therein

17. Shatashvili, S.L.: Correlation Functions in the Itzykson-Zuber Model. Commun. Math. Phys. 154, 421 (1993)

18. Verbaarschot, J.J.M., Weidenmüller, H.A., Zirnbauer, M.R.: Grassmann Integration in Stochastic Quantum Physıcs: The Case of Compound-Nucleus Scattering. Phys. Rep. 129, 367 (1985)

Note added in proof. In the first derivation of the supersymmetric version of the Harish-Chandra-ItzyksonZuber formula (J. Math. Phys. 32, 336 (1991)) I omitted the eigenvalue exchange terms because further integration over the eigenvalues was required. The full result was first published in J. Alfaro, R. Medina, L.F. Urrutia, J. Math. Phys. 36, 3085 (1995). The latter article and my present article were received by the Journal of Mathematical Physics and Communications in Mathematical Physics, respectively, within one week.

\section{Communicated by M. Jimbo}

This article was processed by the author using the $\mathrm{LTT}_{\mathrm{E}} \mathrm{X}$ style file pljourl from Springer-Verlag. 